CARACTERIZACIÓN DE LOS SISTEMAS INTEGRADOS DE GESTIÓN - HSEQ- EN CALIDAD EN LAS EMPRESAS DE SERVICIOS PETROLEROS EN EL DEPARTAMENTO DEL HUILA

\title{
CARACTERIZACIÓN DE LOS SISTEMAS INTEGRADOS DE GESTIÓN - HSEQ- EN CALIDAD EN LAS EMPRESAS DE SERVICIOS PETROLEROS EN EL DEPARTAMENTO DEL HUILA
}

\section{QUALITY CHARACTERIZATION OF THE HSEQ MANAGEMENT INTEGRATED SYSTEMS IN THE OIL SERVICE COMPANIES IN HUILA DEPARTMENT}

\author{
Hernando Gil Tovar*, Estefannya Ramírez Delgado**, \\ Yolimber Ochoa Guevara***
}

\begin{abstract}
*Tutor del Semillero Uscogestión. Director del grupo de investigación PYMES y docente tiempo completo de la Universidad Surcolombiana, Neiva $(H)$, Colombia.

** Administrador de Empresas, Universidad Surcolombiana, Neiva $(H)$, Colombia. Integrante del semillero Uscogestión del grupo de investigación PYMES de la Universidad Surcolombiana. Email: estefannyaramirez.d@gmail.com

***Administrador de empresas, Universidad Surcolombiana, Neiva $(\mathrm{H})$, Colombia. Integrante del semillero Uscogestion del grupo de investigación PYMES de la Universidad Surcolombiana. Email: yolimber.07@gmail.com
\end{abstract}

Dirección para recibir correspondencia: hergil@usco.edu.co 
CARACTERIZACIÓN DE LOS SISTEMAS INTEGRADOS DE GESTIÓN - HSEQ- EN CALIDAD EN LAS EMPRESAS DE SERVICIOS PETROLEROS EN EL DEPARTAMENTO DEL HUILA

\section{RESUMEN}

OBJETIVO: Caracterizar los sistemas integrados de gestión HSEQ, focalizados en el sistema de gestión de calidad, de las empresas que prestan servicios a las compañías petroleras del departamento del Huila.

MATERIAL Y MÉTODO: Las fuentes primarias fueron 19 empresas seleccionadas mediante muestreo por conveniencia, a las cuales se les aplicó una encuesta. Las fuentes secundarias fueron la consulta de artículos, libros, base de datos, revistas y periódicos; conceptuando temas tales como: la Calidad, Sistemas de Gestión, Sistema de Gestión de la Calidad (SGC), el Sistema de Gestión Ambiental (SGA) y el Sistema de Seguridad y Salud en el Trabajo (SST), pertinentes a la comprensión del objeto de estudio. La metodología utilizada es descriptiva con enfoque cuantitativo y un método deductivo.

RESULTADOS: La investigación obtuvo que la integralidad de los sistemas de gestión permite tener empresas del sector petrolero y su cadena de prestadores de servicios, comprometidos con la implementación y actualización de políticas, verificación de requisitos y normatividades, innovación en cada uno de sus procesos en aras de tener un talento humano comprometido con las buenas prácticas ambientales, laborales y personales.

CONCLUSIONES: Implementar el sistema de gestión de calidad en las organizaciones requiere contar con sinergia en cada etapa del proceso del servicio que ofrecen a las petroleras del departamento del Huila. Por esto es determinante en el proceso de mejora continua contar con personal capacitado para desempeñar las funciones propias de su labor, además de la evaluación de las capacitaciones y la toma de conciencia del personal que está al frente de los procesos de satisfacción del cliente.

PALABRAS CLAVE: HSEQ. Calidad. Sistemas de gestión de calidad. Sistemas de gestión ambiental. ISO. 
CARACTERIZACIÓN DE LOS SISTEMAS INTEGRADOS DE GESTIÓN - HSEQ- EN CALIDAD EN LAS EMPRESAS DE SERVICIOS PETROLEROS EN EL DEPARTAMENTO DEL HUILA

\begin{abstract}
OBJECTIVE: To characterize the HSEQ management integrated systems, focused in the quality management system of the companies that provide service to oil companies of Huila Department
\end{abstract}

MATERIAL AND METHOD: Primary sources were19 enterprises that were selected by convenience sampling to which a survey was applied. The secondary sources were the consultation of articles, books, databases, magazines and newspapers; conceptualizing topics such as: Quality, Management Systems, Quality Management System (QMS), Environmental Management System (EMS), and Occupational Health and Safety System (OHS) relevant to the understanding of the object of study. It was used a descriptive methodology with a quantitative approach and a deductive method.

RESULTS: The research indicates that the integrality of the management systems allows the companies of the oil sector and its chain of service providers to be engaged with the implementation and updating of policies, verification of requirements and regulations, as well as the innovation of each one of their processes for the sake of having a human talent committed to good environmental, labor and personal practices.

CONCLUSIONS: Implementing the quality management system in the organizations requires having synergy at each stage of the service process they offer to oil companies of Huila department. For this reason, in this process of continuous improvement, it is crucial to have trained personnel to perform the functions of their work, besides training evaluations and the awareness of the personnel that is at the forefront of the customer satisfaction process.

KEY WORDS: HSEQ. Quality. Quality management systems. Environmental management systems. ISO. 
CARACTERIZACIÓN DE LOS SISTEMAS INTEGRADOS DE GESTIÓN - HSEQ- EN CALIDAD EN LAS EMPRESAS DE SERVICIOS PETROLEROS EN EL DEPARTAMENTO DEL HUILA

\section{INTRODUCCIÓN}

El presente proyecto, realiza una caracterización de los sistemas integrados de gestión HSEQ en calidad, en las empresas de servicios petroleros del departamento del Huila. Se considera de gran impacto para el sector y la academia, ya que son pocos los estudios realizados en este tema. Se hace una caracterización en las empresas de servicios petroleros para lo cual se realiza una amplia revisión conceptual de conceptos como: calidad, sistema, gestión y sistema de gestión de calidad. Se inicia, igualmente, un análisis de los conceptos de sistemas y de gestión, provenientes de autores como Durán (1992), Ureña (1998), Bertalanffy (1986), Ramírez (2002, p. 2), Bustamante \& Aguilar (2013), Zabaleta (2003, p. 118), Cedaro \& Cristaldo (2009), García Dousat, Reyes Jardinez, \& Javier Carballo (2009), Delgado \& Castro (2008) entre otros; para poder llegar al concepto de sistemas de gestión de calidad (Durán, 1990) y HSEQ como sistema de gestión de calidad (Herrera, 2008), (A. V. Feigenbaum, 1983; M. S.), (Feigenbaum \& Pollock, 1999), (Muñoz, 2004), (Ardila, 2012) en donde especifican qué es un sistema de gestión HSEQ en calidad.

El objetivo principal de la investigación fue el de caracterizar los sistemas integrados de gestión -HSEQ- en calidad en el sector de servicios petroleros del departamento del Huila. La metodología utilizada fue cuantitativa y descriptiva, cuyo diseño muestral estuvo conformado por 19 empresas elegidas mediante muestreo por conveniencia, cuyo universo estuvo integrado por las 59 empresas de servicios petroleros ubicadas en el departamento del Huila, teniendo como instrumento para la recolección de información un instrumento (encuesta) para la caracterización de HSEQ en calidad, de las empresas prestadoras de servicios petroleros en el departamento del Huila.

Como resultado del proceso investigativo, el presente estudio llega a la conclusión de que en el sector de servicios petroleros los sistemas de gestión implementados son: Sistema de Gestión de Calidad (SGC), Sistema de Gestión Ambiental (SGA) y el Sistema de Seguridad y Salud en el Trabajo (SST), evidenciando la constante actualización y evaluación las políticas de sistemas integrados. Entre los lineamientos del SGC, se destacan la secuencia en interacción de los procesos como criterios de funcionamiento; el control de los procesos como verificación a las inspecciones que se llevan a cabo de manera interna, el monitoreo a los procesos, la medición del servicio ofrecido y el control a las no conformidades las cuales son reguladas por la búsqueda constante de la efectividad operativa. 
CARACTERIZACIÓN DE LOS SISTEMAS INTEGRADOS DE GESTIÓN - HSEQ- EN CALIDAD EN LAS EMPRESAS DE SERVICIOS PETROLEROS EN EL DEPARTAMENTO DEL HUILA

\section{Fundamentación teórica}

La Ley colombiana 872 de diciembre 30 de 2003 crea el Sistema de Gestión de Calidad en los entes estatales como herramienta de gestión sistemática y transparente que permite dirigir y evaluar el desempeño institucional, en términos de calidad y satisfacción social (SENA, 2005). Yáñez (2008, p. 1) lo define como una forma de trabajar, mediante la cual una organización asegura la satisfacción de las necesidades de sus clientes, para lo cual planifica, mantiene y mejora continuamente el desempeño de sus procesos, bajo un esquema de eficiencia y eficacia que le permite lograr ventajas competitivas.

Los beneficios obtenidos mediante la aplicación de este sistema son:

\section{Tabla 1}

Beneficios en la aplicación del Sistema de Gestión de Calidad

1. Mejora continua de la calidad de los productos y servicios que ofrece.

2. Atención amable y oportuna a sus usuarios.

3. Transparencia en el desarrollo de los procesos.

4. Asegurar el cumplimiento de sus objetivos, en apego a leyes y normas vigentes.

5. Reconocimiento de la importancia de sus procesos e interacciones.

6. Integración del trabajo, en armonía y enfocado a procesos.

7. Adquisición de insumos acorde con las necesidades.

8. Delimitación de funciones del personal.

9. Mejores niveles de satisfacción y opinión del cliente.

10. Aumento de la productividad y eficiencia.

11. Reducción de costos.

12. Mejor comunicación, moral y satisfacción de trabajo.

13. Una ventaja competitiva y un aumento en las oportunidades de ventas.

Fuente: Sistema de gestión de calidad en base a la norma ISO 9001 (Yañez, 2008, p. 2).

En el desarrollo de la investigación se precisaron conceptos claves, tales como: 
CARACTERIZACIÓN DE LOS SISTEMAS INTEGRADOS DE GESTIÓN - HSEQ- EN CALIDAD EN LAS EMPRESAS DE SERVICIOS PETROLEROS EN EL DEPARTAMENTO DEL HUILA

\section{Calidad}

Por los elementos que convergen en los sistemas Integrado de Gestión -HSEQ-, hablar de calidad es inevitable. Se trata de un término en principio estrechamente ligado a la supervisión de los productos por parte de los consumidores, que evolucionó en parte gracias a los aportes de la cultura japonesa. Con el tiempo la aplicación de métodos estadísticos permitió controles más estrictos con menores costos, y como segunda medida, la fiabilidad de los modelos y fórmulas, diseños y factores de seguridad, con el fin de reducir los procesos y costos de fabricación (Ureña López, 1998).

Otro aspecto relevante lo trabaja Mena Hidalgo (2012) como el satisfacer plenamente e ir más allá de las necesidades y requerimientos de los clientes como tarea que todos, dentro de la organización, tienen que lograr, sin mencionar que se generaron una serie de prácticas gerenciales como el Kaizen, las 5-S y la reingeniería de los procesos de negocios y Círculos de Control de Calidad (CCC). Todas en torno a la filosofía del círculo Deming: Planear-hacerverificar-actuar (Aldana de Vega, 2010).

Cabe resaltar, que la necesidad de producir con calidad se hizo vital para el éxito en una organización dada la importancia del cliente y sus expectativas (Ureña López, 1998).

\section{Sistemas}

Los sistemas se encuentran estrechamente relacionados con el trabajo de Ludwing Von Bertalanffy. La idea de este autor surge por la falta de conceptos, que le impiden estudiar los sistemas vivos y que se debería constituir un mecanismo de integración entre las ciencias naturales y sociales para resolver los problemas que presentaban estos (Bertalanffy, 1976).

La Teoría General de Sistemas puede definirse como una forma ordenada y científica de aproximación y representación del mundo real, y simultáneamente, como una orientación hacia una práctica estimulante para formas de trabajo trans-disciplinario y que resalta por su perspectiva integradora (Arnold Cathalifaud \& Osorio, 1998).

\section{Gestión}

La gestión tiene dos ópticas diferentes que la llevan a buscar una filosofía de acciones que procuren el cambio, tal es el caso de los diferentes niveles de gestión en la responsabilidad, comportamiento e importancia organizacional (Cristaldo, 2009). GIL-TOVAR H., RAMÍREZ-DELGADO E., OCHOA-GUEVARA Y. 
CARACTERIZACIÓN DE LOS SISTEMAS INTEGRADOS DE GESTIÓN - HSEQ- EN CALIDAD EN LAS EMPRESAS DE SERVICIOS PETROLEROS EN EL DEPARTAMENTO DEL HUILA

La implementación de un sistema de gestión eficaz ayuda a gestionar los riesgos sociales, medioambientales y financieros (Zapata \& Barrientos, 2013); mejorar la efectividad operativa; reducir costos; aumentar la satisfacción de clientes y partes interesadas; proteger la marca y la reputación; lograr mejoras continuas, además de otras ventajas (Cortés \& Ardila, 2012). Por otra parte, la gestión sistémica basada en procesos contribuye a observar la gestión como un sistema abierto dentro de un sistema más complejo y dinámico en constante interacción con su medio externo. (Delgado \& Castro, 2008). Los sistemas de gestión de la calidad permitieron abordar la gestión de la calidad, desde la segundad mitad del siglo XX hasta la actualidad, siendo de gran interés por plantear temas de planeación, control, aseguramiento y Mejoramiento (Herrera Mendoza, 2008).

"Un sistema de calidad es la estructura funcional de trabajo acordaba en toda la empresa, documentada con procedimientos integrados técnicos y administrativos efectivos, para guiar las acciones coordinadas de la fuerza laboral, las máquinas y la información de la empresa de una forma eficiente, eficaz y más práctica, para asegurar la satisfacción del cliente con la calidad y costos económicos de calidad". (Herrera Mendoza, 2008, p. 11).

Las normas ISO, aceptadas internacionalmente como la "International Organization for Standardization" (ISO) (Montes \& Fuentes, 2005), comprende requisitos para el establecimiento de sistemas de calidad para las diferentes organizaciones con el fin de implementar sistemas de gestión de calidad de manera eficaz; entre ellas se cuenta la ISO 9000, 9001, 9004 y la 19001 (Herrera Mendoza, 2008). Dichas normas permiten controlar las actividades de explotación de recursos, cuidando el medio ambiente y satisfaciendo al cliente, en el caso particular de la industria petrolera.

\section{HSEQ como sistema de gestión de calidad}

El ciclo Deming, permite que el producto o servicio se ponga al nivel de los estándares internacionales, brindando de paso ventajas competitivas a la empresa con procesos de gestión certificados. Latinoamérica no es ajena a esta carrera por la calidad, que es percibida como garantía; "la certificación es una fuente económica, ya que reduce el número de las auditorias de los contratistas" (Muñoz Santos, 1994). 
CARACTERIZACIÓN DE LOS SISTEMAS INTEGRADOS DE GESTIÓN - HSEQ- EN CALIDAD EN LAS EMPRESAS DE SERVICIOS PETROLEROS EN EL DEPARTAMENTO DEL HUILA

Entre los procesos más conocidos para el manejo de procesos el más común es el Sistema de Gestión de Calidad (Quality Management Systems-QMS), seguido por el Sistema de Gestión de Medio Ambiente (Environmental Management System-EMS) y el Sistema de Seguridad e Higiene en el Trabajo (Occupational Safety and Health System-OSH), sin que se haya podido desarrollar un efectivo Sistema Integrado de Gestión.

Es de resaltar, la integración que permite la normatividad entre la ISO 14001 (Gestión Ambiental) y los estándares de la serie ISO 9000 (Gestión de Calidad). Así mismo, la cultura alrededor del HSE (Health- Safety- Environment) y la utilidad de la autorregulación en la gestión integral de los componentes, como lo son; la Seguridad Industrial (SI), Salud Ocupacional (SO) y el Medio Ambiente o Gestión Ambiental (MA, GA). La industria petrolera adoptó estos lineamientos normativos en su estructura interna, pero haciendo exigible a sus contratistas la reglamentación de sus propuestas o licitaciones bajo parámetros de HSE (Peña Mesa, 2015).

En 1996, el British Standards Institution (BSI), publica el primer documento de aplicación internacional de sistemas de gestión de seguridad y salud ocupacional, la norma BS 8800 (Hempstead, 1996). Desde ese entonces hasta nuestra actualidad recobra importancia la implantación de sistemas de gestión que interactúen con su medio y de brindar condiciones de seguridad plenas a sus empleados en casa una de las organizaciones no solo de tipo industrial sino a su vez de comercio y servicios.

\section{MATERIAL Y MÉTODO}

El estudio realizado en el departamento del Huila fue de carácter cuantitativo y el instrumento de recolección empleando, la encuesta (Alaminos \& Castejón, 2006). Este estudio se ocupó de la caracterización de los sistemas integrados de gestión: calidad, medio ambiente y seguridad y salud en el trabajo, focalizados en lineamientos de calidad.

El método de investigación es deductivo, la población está conformada por las empresas prestadoras de servicios petroleros en el departamento del Huila y el formulario de recolección de información está basado en los enfoques teóricos de la ISO 9001-2008.

Se empleó el muestreo por conveniencia dadas las condiciones coyunturales de inestabilidad financiera, baja cotización internacional del crudo del petróleo y su impacto a nivel nacionalregional del sector de petrolero y las empresas prestadoras de servicios en el año 2015. Este tipo de muestreo se establece a juicio de los investigadores donde se seleccionaron 19 GIL-TOVAR H., RAMÍREZ-DELGADO E., OCHOA-GUEVARA Y. 
CARACTERIZACIÓN DE LOS SISTEMAS INTEGRADOS DE GESTIÓN - HSEQ- EN CALIDAD EN LAS EMPRESAS DE SERVICIOS PETROLEROS EN EL DEPARTAMENTO DEL HUILA

empresas de entre un total de 59 que prestan sus servicios a empresas petroleras en el departamento del Huila (Cámara de Comercio de Neiva, 2014). Dentro de las empresas prestadoras de servicios en la industria de hidrocarburos muchas de ellas hacen parte de alianzas internacionales en Colombia.

Se determinó aplicar un cuestionario estructurado de veinte preguntas al encargado del área de calidad de la cada una de las 19 empresas facilitadoras de la información, basado en los enfoques teóricos por la ISO 9001-2008, que establece parámetros de calidad para las organizaciones permitiendo evaluar su relación con el medio ambiente, seguridad ocupacional y seguridad industrial comprendiendo su integralidad.

Dicho instrumento, fue avalado por un panel de experto de la Universidad Surcolombiana, además se realizó una prueba piloto para validar su pertinencia en el desarrollo de los objetivos. Los datos fueron procesados en el programa estadístico SPSS 21, el cual permitió ilustrar los gráficos de cada una de las características de las empresas prestadoras de servicios petroleros en el departamento del Huila.

Como fuentes secundarias de información se utilizaron diferentes bases de datos bibliográficos como Redalyc, artículos pertinentes a los sistemas integrados de gestión, calidad, desarrollo organizacional; además de fuentes estadísticas como las del Departamento Administrativo Nacional de Estadística (DANE, 2014).

Otras de las fuentes utilizadas fueron artículos como la Revista de FEDESARROLLO 2013 Y 2014 (Castro, Zuleta, Alandette, Fonseca, \& Martínez, 2012), aportes muy valiosos a la hora de caracterizar el sector de servicios petroleros en el país y la importancia para el departamento del Huila. Datos bibliográficos como libros de calidad y teorías sobre la evolución del concepto de calidad por los japoneses, la calidad total (Fukui et al., 2003), el Kaizen, las 5-S, la reingeniería de los procesos de negocios y círculos de control de calidad, otros autores citados como: Edward W. Deming -Planear-hacer-verificar-actuar, Bertalanffy sobre la Teoría General de Sistemas y los lineamientos de la Norma ISO 9001, ISO 14001 Y OSHAS 18001.

\section{RESULTADOS}

La investigación da como resultados que el $84 \%$ de las empresas encuestadas está implementando todo el sistema de gestión HSEQ y el 16\% calidad y seguridad industrial y salud ocupacional. Asimismo, el 52\% de estas empresas se encuentran certificadas con International GIL-TOVAR H., RAMÍREZ-DELGADO E., OCHOA-GUEVARA Y. 
CARACTERIZACIÓN DE LOS SISTEMAS INTEGRADOS DE GESTIÓN - HSEQ- EN CALIDAD EN LAS EMPRESAS DE SERVICIOS PETROLEROS EN EL DEPARTAMENTO DEL HUILA

Certification and Training (IC\&T S. A) y Bureau veritas certification y el $48 \%$ con el Consejo Colombiano de Seguridad COTECNA, certificadores de servicios Ltda., Instituto Colombiano de Normas Técnicas y Certificación (Icontec), servicio Nacional de Aprendizaje (SENA) y American Petroleum Institute (API).

El 32\% de las empresas prestadoras de servicios petroleros en el departamento del Huila, tiene una secuencia e interacción de sus procesos, además de criterios de funcionamiento y control de estos, el 22\% implementa un sistema de gestión de calidad, dispone de recurso e información para el funcionamiento; por otra parte, el $46 \%$ lleva uno de los elementos para poder lograr sistemas efectivos y mejorar la calidad de productos o servicios, en referencia a la verificación de la satisfacción de los clientes.

El 42\% de las empresas del estudio utilizan un instrumento de recolección de datos para saber qué tan satisfechos están los clientes con la prestación del servicio, mientras el 32\% utiliza para este fin los informes, quejas y opiniones, y el $26 \%$ utiliza algún otro método para la verificación de la satisfacción de sus clientes.

El 16\% de las empresas socializa al personal por cada departamento de calidad, gerentes de departamento o líderes de procesos, en tanto que el 26\% hace una evaluación con la junta de socios o los jefes de departamento de calidad.

El 21\% de las empresas logran tener un sistema eficiente y eficaz con la implementación del SGC teniendo en cuenta la planificación del sistema, mientras el 11\% lo hace por medio de controles de documentos que generan información y evidencias para que estén disponibles en todo momento para acciones correctivas y preventivas y el $68 \%$ logra la eficiencia y eficacia implementando algún elemento del sistema de gestión de calidad.

El 32\% de las actividades de las empresas del estudio causa un impacto negativo en los suelos.

El 21\% impacta en el consumo de energía y el $47 \%$ restante causa daño al suelo, la atmosfera, las cuencas hídricas, flora y fauna. Asimismo, el 32\% de las empresas recicla y/o realiza un manejo adecuado de sus residuos, implementa programas de gestión ambiental, realiza mantenimiento preventivo, utiliza la matriz de aspectos e impactos ambientales, planes de prevención, elementos y actividades de cumplimiento legal. El 22\% implementa programas de limpieza en las cuencas hídricas y manejo de residuos, en tanto que el $46 \%$ utiliza uno de los aspectos mencionados. 
CARACTERIZACIÓN DE LOS SISTEMAS INTEGRADOS DE GESTIÓN - HSEQ- EN CALIDAD EN LAS EMPRESAS DE SERVICIOS PETROLEROS EN EL DEPARTAMENTO DEL HUILA

Mientras las empresas en las que se participa en prácticas y procedimientos para la prevención de los riesgos no superan el $32 \%$, el $21 \%$ señaliza las áreas, capacita a sus empleados en manejo de extintores y primeros auxilios, como forma preventiva para cualquier riesgo laboral. El 47\% realiza alguna práctica o procedimiento para la prevención del riesgo dentro de su organización.

En la actual coyuntura, los recortes de personal en el sector se han generalizado y afectado a $37 \%$ de las empresas prestadoras de servicios petroleros. Otro aspecto importante es el seguimiento a los reclamos como medida para minimizar las quejas sobre eficiencia operativa, la cual es empleada por el 37\% de las empresas seleccionadas para el estudio, mediante buzón de sugerencia $16 \%$, y encuestas de satisfacción el $26 \%$.

Procesos como la verificación a las inspecciones que se llevan a cabo de manera interna, el monitoreo a los procesos, medición del servicio ofrecido y control a las no conformidades suman $54 \%$ en las empresas de servicios petroleros del departamento del Huila (Diaz Ropero, 2014). A su vez, estas empresas consideran que la evaluación de pos-entrega del servicio y las especificaciones del servicio por los clientes, con 42\% y $16 \%$, respectivamente. Por tanto, son necesarias a la hora de ofrecer un servicio con altos estándares de calidad que busquen satisfacer los requerimientos que la empresa contratante necesita.

En cuanto al seguimiento de las no conformidades, el 11\% de las empresas lo hace con igual porcentaje la determinación e implementación de acciones necesarias y la importancia de determinar las causas potenciales de las no conformidades, evaluar la necesidad de las acciones para impedir que los problemas se repitan y el seguimiento a las acciones correctivas. El 32\% de los encuestados desempeña sus funciones en su puesto de trabajo, 33\% considera que la capacitación continua, su evaluación, la toma de conciencia y registro de los compromisos son determinantes para mantener al personal actualizado y que participe de los procesos de gestión de las organizaciones prestadoras de servicios petroleros.

El 37\% de las empresas resalta que en la potencialización de los procesos de innovación a través del liderazgo se debe contar con recursos y promover espacios para la investigación, el desarrollo y la tecnología, mientras que un 16\% considera que tener procesos de innovación organizacional es algo que se debe gestionar con planes tanto del sistema de gestión de calidad como de los procesos que se llevan a cabo en casa empresa. Otra característica para resaltar es la inmersión en procesos de innovación y mejora continua de los procesos (11\%), 
CARACTERIZACIÓN DE LOS SISTEMAS INTEGRADOS DE GESTIÓN - HSEQ- EN CALIDAD EN LAS EMPRESAS DE SERVICIOS PETROLEROS EN EL DEPARTAMENTO DEL HUILA

junto a la formación y capacitación (6\%), consideradas claves para mantener un sistema de gestión vigente.

Las empresas que prestan servicios petroleros identifican las medidas de prevención, seguridad industrial y salud ocupacional como obligatorias en un $100 \%$. Por otra parte, el $58 \%$ de los encuestados son hombres encargados de los departamentos de calidad y $42 \%$ mujeres, de los cuales el 68\% tiene edades comprendidas entre los 25 y 40 años, el otro $32 \%$ oscila entre los 40 y 55 años.

\section{CONCLUSIONES}

En la investigación realizada sobre la caracterización de los sistemas integrados de gestión HSEQ- en calidad, en el sector de servicios petroleros se encontró que los sistemas de gestión implementados son: SGC, SGA y el SST, evidenciando la constante actualización y evaluación las políticas de sistemas integrados.

Entre los lineamientos del SGC, se destacan la secuencia en interacción de los procesos como criterios de funcionamiento, el control de los procesos como verificación a las inspecciones que se llevan a cabo de manera interna, el monitoreo a los procesos, la medición del servicio ofrecido y el control a las no conformidades. Dichos manejos a las no conformidades están dados por la efectividad operativa (37\%).

Los beneficios que enmarca estar implementando un sistema integrado de gestión se caracteriza por el compromiso y la participación de todas las personas que trabajan para la empresa (21\%). Otra característica para resaltar es el compromiso con los procesos de innovación y mejora continua de los procesos (11\%), además de exaltar la formación y capacitación (6\%) como determinantes para mantener un sistema de gestión vigente.

La implementación del sistema de gestión de calidad en las organizaciones requiere contar con sinergia en cada etapa del proceso del servicio que ofrecen a las petroleras del departamento del Huila. Por esto, es determinante en el proceso de mejora continua contar con personal capacitado para desempeñar las funciones propias de su labor, además de la evaluación de las capacitaciones y la toma de conciencia del personal que está al frente de los procesos de satisfacción del cliente. La mayoría del personal encargado de las labores en los departamentos de calidad son personas jóvenes participes del proceso de innovación y de liderazgo. 
CARACTERIZACIÓN DE LOS SISTEMAS INTEGRADOS DE GESTIÓN - HSEQ- EN CALIDAD EN LAS EMPRESAS DE SERVICIOS PETROLEROS EN EL DEPARTAMENTO DEL HUILA

Es claro que la satisfacción del cliente es el eje central del sistema de gestión de calidad en el que se requiere llevar el control y seguimiento a las no conformidades. Las empresas consideran que evaluar el pos-entrega del servicio y las especificaciones del servicio, son cuestiones necesarias para ofrecer un servicio con altos estándares de calidad.

Se logró evidenciar que las empresas prestadoras de servicios petroleros con más contratos ejecutados son aquellas que cumplen con la totalidad de la normatividad y reglamentación, siendo éste un incentivo adicional más allá de las necesarias medidas de seguridad que estas empresas deben adoptar dadas sus actividades que en algunos casos son de riesgo para sus colaboradores y ambiente.

\section{REFERENCIAS BIBLIOGRÁFICAS}

Alaminos, A., \& Castejón, J. L. (2006). Elaboración, análisis e interpretación de encuestas, cuestionarios y escalas de opinión. Alicante, España: Marfil.

Aldana de Vega, L. Á. (2010). Administración por calidad. Colombia: Alfaomega.

Arnold Cathalifaud, M., \& Osorio, F. (1998). Introducción a los conceptos básicos de la teoría general de sistemas. Cinta de Moebio, 3.

Bertalanffy, L. (1976). Teoría general de los sitemas. México: Fondo de cultura económica.

Cámara de Comercio de Neiva. (2014). Base de datos empresas del Sector de hidrocarburos. Recueperado de https://ccneiva.org/servicios-empresariales/informes-economicos/

Castro, F., Zuleta, L. A., Alandette, J. F., Fonseca, S. M., \& Martínez, S. (2012). Dinámica regional del sector de bienes y servicios petroleros en Colombia. Colombia: Fedesarrollo.

Cortés, D. M., \& Ardila, A. V. (2012). Metodología para la implementación de un sistema integrado de gestión con las normas ISO 9001, ISO 20000 e ISO 27001. Bogotá: Universidad EAN. Facultad de Ingeniería. Modalidad Trabajo de Grado.

Cristaldo, K. C.-I. P. (2009). Sistemas de gestión.

Dane, D. A. N. d. E. (2014). Boletín informativo. Bogotá D.C: DANE. 
CARACTERIZACIÓN DE LOS SISTEMAS INTEGRADOS DE GESTIÓN - HSEQ- EN CALIDAD EN LAS EMPRESAS DE SERVICIOS PETROLEROS EN EL DEPARTAMENTO DEL HUILA

Delgado, M., \& Castro, E. (2008). La pertinencia social en el sistema de gestión del proceso creador de conocimiento científico en las organizaciones universitarias. Telos, 10(1).

Diaz Ropero, V. A. (2014). Supervisión del montaje de elementos mecánicos y control de calidad en la empresa profesionales técnicos SAS en el municipio de Tesalia Huila. Universidad Francisco de Paula Santander Ocaña.

Durán, M. U. (1990). La revolución industrial por la calidad: los japoneses. Estratos (18), pp. 5661.

Feigenbaum, A. V. (1983). Total quality control. New York: McGraw Hill.

Feigenbaum, M. S., \& Pollock, M. L. (1999). Prescription of resistance training for health and disease. Medicine and science in sports and exercise, 31, pp. 38-45.

Fukui, R., Honda, Y., Inoue, H., Kaneko, N., Miyauchi, I., Soriano, S., \& Yagi, Y. (2003). Manual de administración de la calidad total y círculos de control de calidad. Volumen I. Recuperado de http://www.inacal.org.uy/files/userfiles/file/VII_\%20ManualACTyCCC.pdf

García Dousat, Y., Reyes Jardinez, L., \& Javier Carballo, C. (2009). ¿ Por qué la importancia de implementar Sistemas de Gestión por Competencias en nuestras organizaciones? Ciencias Holguín, 15(2).

Herrera Mendoza, M. (2008). Diseño de un sistema de gestión de la calidad para una microempresa.

Mena Hidalgo, N. I. (2012). Modelo de gestión de calidad y la mejora contínua de los servicios en línea en la Comercializadora Valdiviezo Venegas Cía. Ltda. de la ciudad de Latacunga.

Montes, F. J. L., \& Fuentes, M. d. M. F. (2005). Gestión de la calidad empresarial: Fundamentos e implantación: España: Pirámide.

Muñoz Santos, J. R. (1994). La gestión integrada: calidad, seguridad y medio ambiente: España: SERFOREM, SL.

Peña Mesa, D. A. (2015). Diseño de un Sistema de Gestión en Seguridad, Salud en el Trabajo y Protección Ambiental para la empresa Kaysen Soluciones SAS bajo los parámetros de la guía para contratistas RUC®.

GIL-TOVAR H., RAMÍREZ-DELGADO E., OCHOA-GUEVARA Y.

MAYO-AGOSTO 2018. Año 24, Número 69. Págs. 267-281 
CARACTERIZACIÓN DE LOS SISTEMAS INTEGRADOS DE GESTIÓN - HSEQ- EN CALIDAD EN LAS EMPRESAS DE SERVICIOS PETROLEROS EN EL DEPARTAMENTO DEL HUILA

SENA. (2005). Manual de procesos y procedimientos, sistema de gestión de calidad. Recuperado

de repositorio.sena.edu.co/bitstream/11404/3672/1/manual_procesos_procedimientos.pdf

Ureña López, A. E. (1998). Gestión estratégica de la calidad (Tesis doctoral, Universidad de Malaga). Recueperada de http://www.biblioteca.uma.es/bbldoc/tesisuma/16279463.pdf

Yáñez, C. (2008). Sistema de gestión de calidad en base a la norma ISO 9001. Recuperado de: http://internacionaleventos.com/articulos/articulolso. pdf.

Zabaleta, A. T. (2003). Los modelos actuales de gestión en las organizaciones. Gestión del talento, gestión del conocimiento y gestión por competencias. Psicología desde el Caribe, (12).

Zapata, L. F. B., \& Barrientos, S. C. A. (2013). Retos de gestión intercultural en las actividades de internacionalización de microempresas de Medellín. Estudios Gerenciales, 29(129), pp. 456-465. 